\title{
Increasing the Performance of PES-CNTs Mixed Matrix Membrane using Carbon Nanotubes (CNTs) Functionalization
}

\author{
A. Mustafa ${ }^{\text {b }}$, T.D. Kusworo ${ }^{\text {a }}$, Abdullah Busairi ${ }^{\text {a }}$, A.F. Ismail ${ }^{\mathrm{b}}$, and Budiyono ${ }^{\mathrm{a}}$ \\ ${ }^{a}$ Faculty of Engineering, University of Diponegoro, \\ Jl.Prof. Sudharto Tembalang, Semarang, Indonesia.tutukjoko@yahoo.com \\ ${ }^{b}$ Faculty of Chemical Engineering and Natural Resources, \\ University of Technology Malaysia, Skudai Johor Baharu, Malaysia \\ *Corresponding Author: tutukjoko@yahoo.com
}

\begin{abstract}
A new type of mixed matrix membrane consisting of functionalized carbon nanotubes (CNTs) and polyethersulfone (PES) is prepared for biogas purification. PES mixed matrix membrane with and without modification of carbon nanotubes were prepared by a dry/wet phase inversion technique using a pneumatically flat sheet membrane casting machine system. The modified carbon nanotubes were prepared by treating the carbon nanotubes with chemical modification using Dynasylan Ameo (DA) silane agent to allow PES chains to be grafted on carbon nanotubes surface. The results from the FESEM, DSC and FTIR analysis confirmed that chemical modification on carbon nanotubes surface had taken place. Meanwhile, the nanogaps in the interface of polymer and carbon nanotubes were appeared in the PES mixed matrix membrane with unmodified of carbon nanotubes. The modified carbon nanotubes mixed matrix membrane increases the mechanical properties, the productivity and purity of biogas. For PES-modified carbon nanotubes mixed matrix membrane the maximum selectivity achieved for $\mathrm{CO}_{2} / \mathrm{CH}_{4}$ is 36.78

Keywords - Mixed matrix membrane, carbon nanotubes, biogas, gas separation
\end{abstract}

\section{INTRODUCTION}

Off-gas from anaerobic digestion and landfills has significant potential as an alternative energy source. At present it can be used only at the place where it is produced, most often for steam generation. However, the use of biogas still has limitation due to low-quality of biogas containing substantial amounts of $\mathrm{CO}_{2}\left(20-45\right.$ vol\%) and $\mathrm{H}_{2} \mathrm{~S}(0-1 \mathrm{vol} \%)$ in addition to main component $\mathrm{CH}_{4}$ (55-80 vol\%). In addition, biogases are also frequently saturated by water vapour. The presence of $\mathrm{CO}_{2}$ as an incombustible gas reduce its calorific value and make it uneconomical to compress and transport to longer distances [1]. Biogas purified and enriched in methane can be used for household applications, automobile fuel (liquefied) or electricity generation. There is a great need to make biogas transportable. This can be done by compressing the gas in cylinders, what is possible only after removing $\mathrm{CO}_{2}$, $\mathrm{H}_{2} \mathrm{~S}$ and water vapour. There is a lot of potential if biogas could be made viable as a transport vehicle fuel like CNG, by compressing and filling the cylinders in it after scrubbing and drying. Enrichment of methane in biogas to have fuel of higher calorific value can be achieved by removal of carbon dioxide. Elimination of carbon dioxide from the flue gas helps to increase its calorific value as well as to eliminate the greenhouse gas. The carbon dioxide thus generated can be utilised as an effective refrigerant.

Currently, a variety of processes are being used for removing $\mathrm{CO}_{2}$ and $\mathrm{H}_{2} \mathrm{~S}$ from $\mathrm{NG}$ in petrochemical industries. Several basic mechanisms are involved to achieve selective separation of gas constituents. These may include physical or chemical absorption, adsorption on a solid surface, membrane separation, cryogenic separation and chemical conversion. Chemical methods are based on absorption under elevated pressure (in water, $30 \%$ solution of potassium carbonate, solution of monoethyloamine, etc.). Other methods are based on adsorption in which a vital role plays suitable adsorbent material. A very important stage of the biogas upgrading is its purification from $\mathrm{H}_{2} \mathrm{~S}$ traces. The biogas desulphurisation is based on a chemical reaction of $\mathrm{H}_{2} \mathrm{~S}$ with a suitable substance, such as quicklime, slaked lime in solid form, or slaked lime in liquid form. High concentration of $\mathrm{CO}_{2}$ which is present in biogas makes the satisfactory removal of $\mathrm{H}_{2} \mathrm{~S}$ difficult; the $\mathrm{CO}_{2}$ also reacts with the quick and slaked lime and uses it up quickly. At the present, membrane separation processes have emerged during the last two decades. This is due to the fact that membrane separation processes may offer more capital and energy efficiency when compared to the conventional separation processes [2]. In addition, advantage of membrane technology is its simplicity, i.e. no absorbent, which has to be regenerated; it can be easily retrofitted, modularized and scaled-up for several applications [3].

At the present, polymeric membranes dominate the membrane separation field due to the fact that they are well developed and quite competitive in separation performance and economics. Since the separation of gas mixtures depends on the relative solubility and diffusivities of individual components within a particular membrane, no single membrane material is capable of successfully separating all gas mixtures. The choice of membrane material will be dictated by the nature of the gases mixture to be separated. Glassy polymers such as polysulfone, polyethersulfone, polyimide, and ethyl cellulose which are known to have high mechanical stability and desirable inherent transport properties at high temperature are more commonly used [4]. 
Despite concentrated efforts to tailor polymer structure to affect separation properties; current polymeric membrane materials have seemingly reached a limit in the trade-off between productivity and selectivity. Pure polymer membranes are oftentimes shows several limitations as low selectivity, high temperature instability and swelling and decomposition in organic solvents. Hybrid membranes composed organic- inorganic has attracted attention as the future membrane material. Hybrid membranes or mixed matrix membrane concept combines the advantages of high separation capabilities of the molecular sieves and the desirable mechanical properties and economical processing capabilities of polymers [4-6]. Many studies have reported that the separation performance of mixed matrix membrane could be improved by integrating porous or nonporous inorganic filler such as zeolite, silica, carbon molecular sieve and activated carbon. Currently, significant efforts have been devoted in fabrication of mixed matrix membrane by use carbon nanotubes as great potential filler. The properties of polymer nanocomposites containing carbon nanotubes depend on several factors in addition to the polymer: synthetic process used to produce nanotubes; nanotube purification process (if any); amount and type of impurities in the nanotubes; diameter, length, and aspect ratio of the nanotubes objects in the composite (isolated, ropes, and/or bundles); nanotubes orientation in the polymer matrix. Kim et al [7] studied the effect of incorporating of CNTs on polyimidesiloxane matrix on gas separation performance. They observed that the addition of small CNTs to the copolymer matrix will be reducing the permeability helium and hindering the diffusion of nitrogen due to the impermeable properties of CNTs.

Currently, different approaches are reported to improve the dispersion of CNTs in solvents or polymers in order to fabricate the composite mixed matrix nanotubes membrane. Recent reports on the chemical compatibility and dissolution properties of CNTs have promoted a great deal of interests in developing modification and functionalization of their surface. The chemical functionalization of multiwall CNT can be done by using novel silane coupling agents which helps to covalently link polymers. Besides that, treatment by carboxylic acid groups also can be used in this modification but for the strong acid used it can defects on CNTs sidewalls and decrease the aspect ratio of CNTs and other possible surface modification techniques included plasma, thermal and laser ablation [8]. By using silane agents, the coupling process can be accomplished via the chemical reaction between the trialkoxy groups of silane molecules and the hydroxyl groups of silane molecules and the hydroxyl groups on the glass substrates, whereas other functional group of silane molecules, which are generally ethylene, amine, epoxy and thiohydroxy can be remained [9].

Therefore, in the present study, the feasibility fabrication and characterization result of mixed matrix membrane films using carbon nanotubes particles as selective inorganic fillers was investigated. The carbon nanotubes were functionalized using Dynasylan Ameo silane coupling agent. The aim is to get a CNTs linked with the coupling agent having a functional group such as a double bond which can be utilized further for copolymerization. Hence, this reaction product (CNTs silanization) can be chemically explored for improving their compatibility with polymer matrix.

\section{METHODS}

Polyethersulfone was supplied by Solvay Advanced Material (USA). The polymers were dried in a vacuum oven at $120 \mathrm{oC}$ overnight before dope preparation; N-methylpyrrolidinone (NMP) from Merck was used as the solvent due to its low toxicity. The Single wall nanotubes (SWNT) were produced using Thermal Catalytic Chemical Vapour Deposition (CCVD. The particle size of CNTs was about $25.76 \mathrm{~nm}$. In this study, the polymer solution consists of 25 wt $\%$ polymer, $75 \%$ NMP and $1 \mathrm{wt} \%$ nanotubes in the total solid. Flat sheet membrane was prepared according to the dry/wet phase inversion technique. The solution was poured onto a clear, flat and smooth glass plate that was placed on the trolley. Stainless steel support casting knife was used to spread the solution to a uniform thickness by pneumatic force. Carbon nanotubes samples were additionally modified by a silane coupling agent. First, the CNTs were again dried and pre-treated in $\mathrm{H} 2 \mathrm{SO} 4$. Double bonds present in the aromatic nanotube structure were oxidized with a sulfuric acid. This pre-treatment is necessary to create carboxylic acid moieties on nanotubes sidewalls that can react with the silane and also to remove impurities from the supported catalyst. Finally, the modified carbon nanotubes was dehydrated at $110{ }^{\circ} \mathrm{C}$ for 5 hours in a vacuum oven to remove the adsorbed water vapor or other organic vapors before it was ready to be used in preparation of dope solution.

The membrane sheets were coated with highly permeable elastomeric silicone polymer (Sylgard 184 Dow Corning). The membrane coating was done after the uncoated membranes were tested. The intention of coating is to fill any surface pinholes or defects on membrane surface. Membranes were submerged in the $3 \% \mathrm{w} / \mathrm{w}$ solution of silicone in $\mathrm{n}$-hexane for 24 hours and subsequently placed in oven for 3 days at $120 \mathrm{oC}$ to allow curing before permeation testing. A Supra 35 VP Field Emission Scanning Electron Microscopy (FESEM) was used to ensure the asymmetric structure and to determine the dimension of the fibers. The glass transition temperature of each cast film was determined using differential scanning calorimetry (Mettler Toledo DSC 822e). A small piece of membrane or pure polymer sample was first stored under vacuum at $100 \mathrm{oC}$ for 24 hours to remove adsorbed water; then weighed and placed into aluminium DSC pans. The scanning range was 50- $320 \mathrm{oC}$ with scanning rate of $10 \mathrm{oC}$ min-1 in the first DSC cycle to remove thermal history and then cooled from 320 to $25 \mathrm{oC}$ at the rate of $10 \mathrm{oC}$ min-1; finally the second cycle was carried out with the same procedure.

The permeation test involved the use of gas permeation cell in which the membrane was placed on a sintered metal plate and pressurized at the feed side. Gas permeation rates were measured by a constant pressure system using a soap bubble flow meter. Pressure normalized gas permeation flux or permeance for gas $\mathrm{I}$, (P/l)I, in (GPU), can be calculated as follows:

$$
\left(\frac{P}{l}\right)_{i}=\frac{Q_{i}}{A \Delta p}
$$

where Qi is the volumetric flow rate of gas $\mathrm{i}$, is pressure difference across membrane ( $\mathrm{cmHg}), \mathrm{A}$ is membrane affective surface area $\left(\mathrm{cm}^{2}\right)$ and 1 is membrane skin thickness $(\mathrm{cm})$. The ideal separation factor can be calculated by using equation below: 


$$
\left(\frac{P}{l}\right)_{i}=\frac{Q_{i}}{A \Delta p}
$$

\section{RESULTS AND DISCUSSIONS}

In order to further investigate the effect of silanization of CNTs on the dispersion of CNTs in mixed matrix membrane, careful FESEM inspections were carried out. The FESEM micrographs of the cross-sectional and the surface of the neat PES, PES-unmodified nanotubes and PES-modified nanotubes mixed matrix membranes are shown in Figures 1-3.

As shown in Figures 1-3, structure of nanotubes composite membranes showing finger like structure which consisted of a dense skin layer supported by a spongy porous substructure. The unmodified carbon nanotubes particle seemed to good adhere with polymer matrix as shown in Figure 2. The smooth surface of the unmodified nanotubes-PES mixed matrix membrane might be due to the shape of the carbon nanotubes particles appeared to be oblong, therefore no sharp edges that could easily perforated the active surface skin. However, in the case of unmodified carbon nanotubes, the unselective voids appeared indicated that the carbon nanotubes did not completely adhered on the surface of polymer matrix. The small surface ruptures and aggregation are also occurred on the mixed matrix membrane with unmodified carbon nanotubes as shown in Figure $2 b$.

The cross-section and surface of PES-modified CNTs using $15 \mathrm{wt} \%$ of silane are shown in Figure 3(a)-(b). The porous substrate of the fabricated membrane and the active skin layer can be seen in Figure 3. Cross-section of porous substrate region for PES modified CNT clearly show the compatibility between modified CNT loading with PES. Moreover, from the cross section can be seen that CNTs clearly act as the filler to in the PES polymer host. Due to the chemical and physical properties of amino silane could be able to enhance the bond strength which provides a stable and strong bond between the modified CNT with PES matrix and finally reduced the presence of voids surrounded on CNT surface. The strong bonding was produced by the adsorption of functional groups of curing agent into amino-functionalized CNTs because of strong interfacial bond, which may lead to the nonstoichiometric balance between epoxy prepolymer and curing agent, thus leading to the inhibition of cross-linking reaction between them and when the content of CNTs is higher, congregation of CNTs because of intrinsic van der Waals forces possibly occurs, leading to bubbles and small aggregates [7]. Therefore, the good compatibility between polymer matrix and carbon nanotubes would finally lead to diminish gas penetrants via unselective voids of carbon nanotubes and simultaneously high gas separation performance of membrane is able to be achieved. Figure 3 had also revealed the presence of some CNT under the skin layer due to the cutting in nitrogen. The smooth surface of carbon nanotubes might also induce to enhance the adhesion between the nanotubes and the host of polymer.

The Differential Scanning Calorimetry has been done to indicate whether the CNT-PES blends were indeed miscible by observing the glass transition temperatures of the blend composition. The $\mathrm{Tg}$ for each membranes was determined from the heat flow versus temperature curve using an onset method. The influence of silane modification on the glass transition temperature of PES-carbon nanotubes mixed matrix membranes are tabulated in Table 1. As shown in Table 1, the glass transition value, the amino-functionalized CNT have increased the glass transition temperature $\mathrm{Tg}$. This can be seen in the effect of $\mathrm{Tg}$ for CNT modification with $15 \mathrm{wt} \%$ silane. The increasing of $\mathrm{Tg}$ in amino-functionalized CNTs MMM is because of the strong interaction between the PES matrix and amino-functionalized membrane which reduce the matrix mobility which will express itself in a shift of $\mathrm{Tg}$ [10] and beside that the diffusion of small molecules can be retarded under high temperature. It can also be seen that compared with neat PES polymer, the Tg of the mixed matrix membranes increased about $5 \mathrm{oC}$ with the incorporating only $1 \mathrm{wt} \%$ modified carbon nanotubes using $15 \mathrm{wt} \%$ silane treatment into the matrix. This phenomenon indicates that the mobility of polymer chains is reduced due to the restriction effect of carbon nanotubes. This result is in agreement with the previous study [11]. Based on Table 1, it can be concluded that the carbon nanotubes surface modification using Dynasylan Ameo silane coupling agent could induced the adherence between polymer matrix and carbon nanotubes particles. Therefore, it can be concluded that modified carbon nanotubes using chemical modification included into PES matrix will be made the composite film stiffer and stronger due to strong interfacial interaction between the nanotubes and PES host matrix. Moreover, with addition the nanotubes the movement of polymer chain in polymer host matrix will be restricted and this phenomenon likes physical cross-linking.

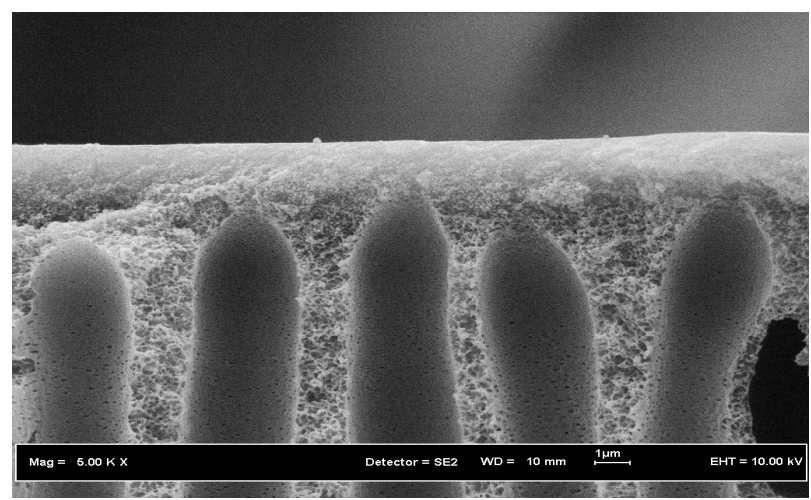

(a)

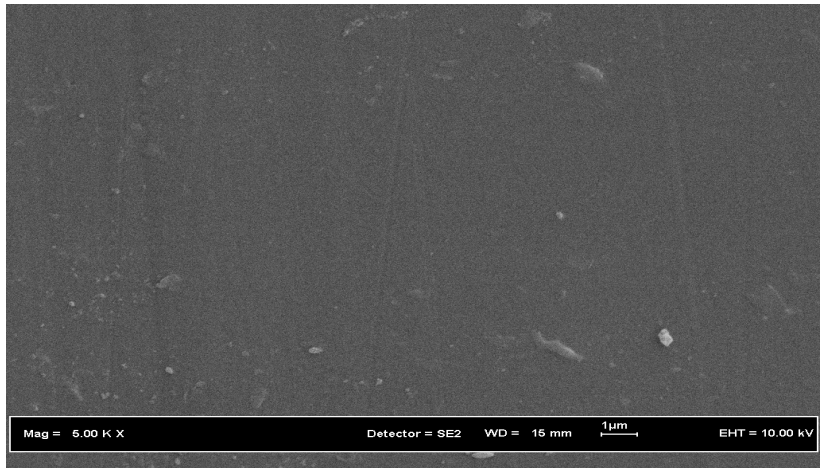

(b)

Figure 1: SEM picture of asymmetric polyethersulfone membrane at the: (a) cross section and (b) outer surface image layer. 


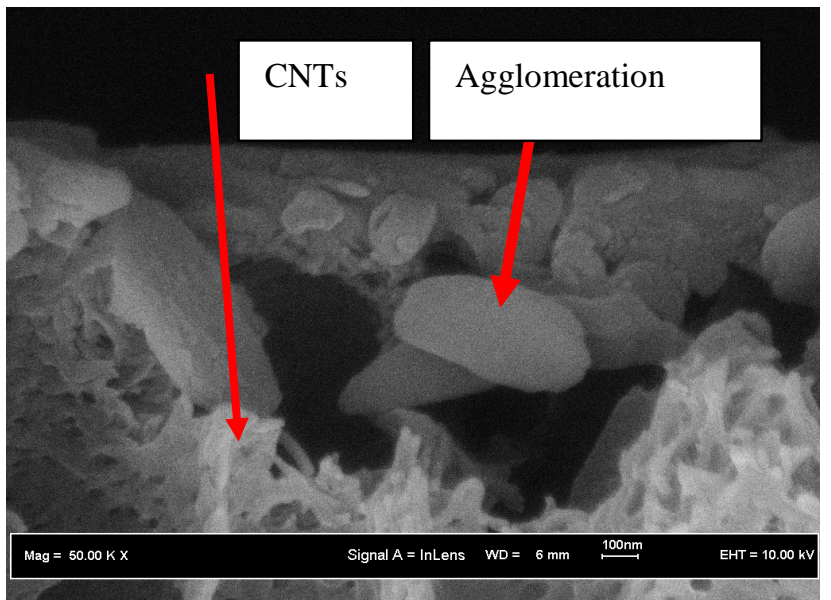

(a)

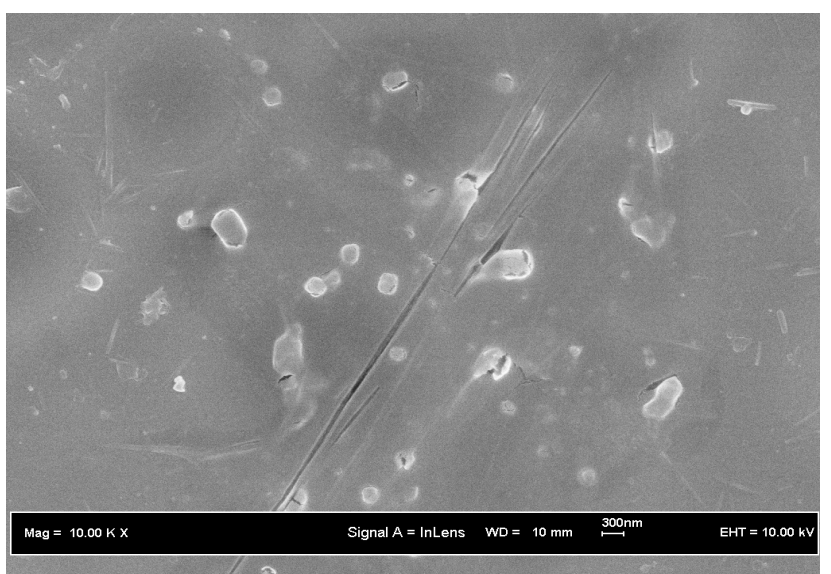

(b)

Figure 2: SEM picture of asymmetric polyethersulfone-unmodified CNTs membrane at the: (a) cross section and (b) outer surface image layer.

TABLE 1

EFFECT OF FUNCTIONALIZATION OF CNTS ON THE GLASS TRANSITION TEMPERATURE OF PES-CNTS MIXED MATRIX

\begin{tabular}{lc}
\multicolumn{2}{l}{ MEMBRANE } \\
\hline Membrane & $\mathbf{T}_{\mathbf{g}}\left({ }^{\mathbf{0}} \mathbf{C}\right)$ \\
\hline Neat PES & 219.05 \\
Untreated CNTs & 221.07 \\
15 wt $\%$ DA silane & 224.65
\end{tabular}

In the gas separation performance, the criteria which is important in determining whether the membrane are classified as good or not was determined by the ability of the membrane to give the high enhancement in gas separation performance than others membrane. Thus, this testing was carried out in order to study the membrane effectiveness due to the effect of chemical modification on carbon nanotubes surface.

The membrane effectiveness in the gas separation performance was determined by the membrane permeability and selectivity for tested biogas purification. In this study, we were used gas sample of $\mathrm{CO}_{2}$ and $\mathrm{CH}_{4}$ to represent the biogas product. The gas permeation properties for each flat sheet carbon nanotubes mixed matrix membrane were measured by using variable pressure constant volume method. The permeability and selectivity for tested gases $\mathrm{CO}_{2} / \mathrm{CH}_{4}$ obtained were presented in Table 2. The Table 2 summarizes the permeability and selectivity data of neat PES, PES unmodified and PES-modified CNT at various silane concentrations.

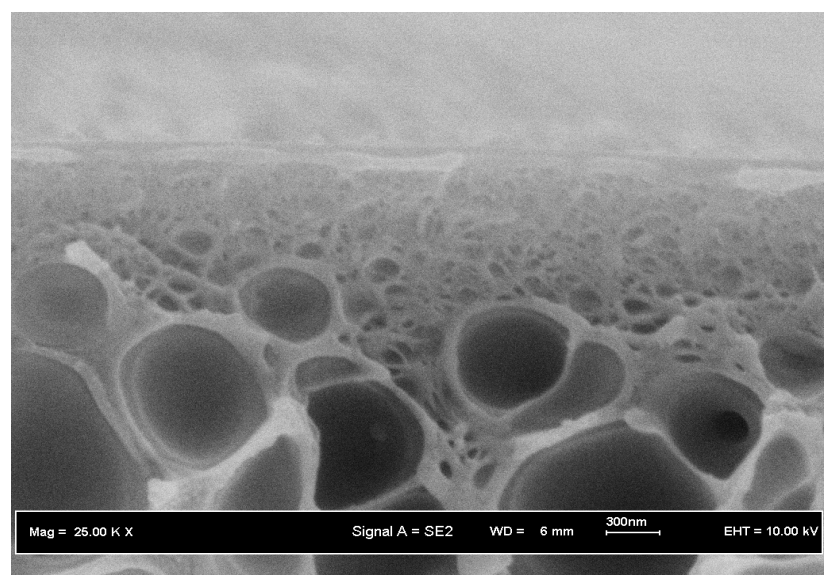

(a)

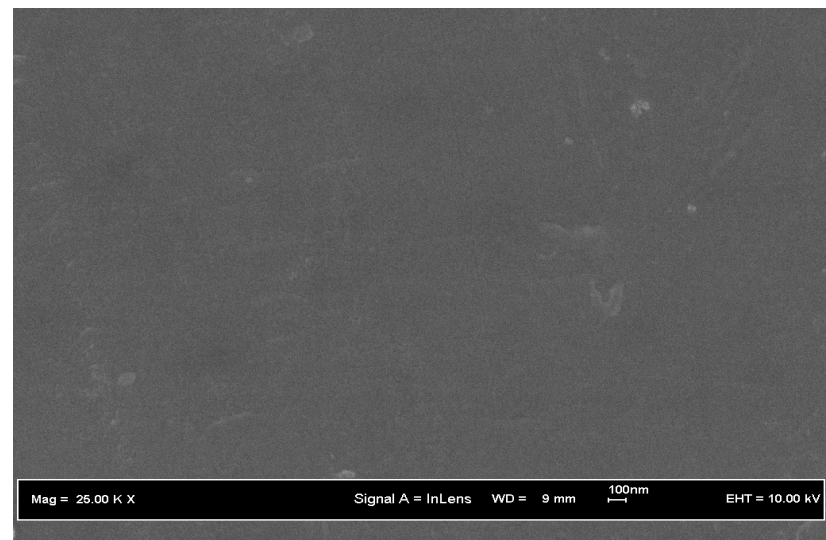

(b)

Figure 3: SEM picture of asymmetric polyethersulfone-modified CNTs membrane at the: (a) cross section and (b) outer surface image layer.

\section{TABLE 2}

EFFECT OF SILANE CONCENTRATION ON THE GAS SEPARATION PERFORMANCE OF POLYETHERSULFONE-CNTS MIXED MATRIX MEMBRANES AT ROOM TEMPERATURE AND 10 BARS

\begin{tabular}{|c|c|c|c|}
\hline \multirow[t]{2}{*}{ Membrane } & \multicolumn{2}{|c|}{$\begin{array}{c}\text { Single gas permeance } \\
\text { (GPU) }\end{array}$} & \multirow{2}{*}{$\begin{array}{l}\text { Selectivity } \\
\mathrm{CO}_{2} / \mathrm{CH}_{4}\end{array}$} \\
\hline & $\mathrm{CO}_{2}$ & $\mathrm{CH}_{4}$ & \\
\hline Neat PES & 20.36 & 0.70 & 28.75 \\
\hline Untreated membrane & 25.18 & 1.96 & 12.84 \\
\hline 15 wt $\%$ DA silane & 22.18 & 0.73 & 30.42 \\
\hline
\end{tabular}

Generally, the idea to put inorganic filler into organic polymer was enhanced gas permeability of polymer nanocomposites membranes was due to the disturbed polymer chain packing by the nanofillers. Therefore, the well dispersed and good adherence of carbon nanotubes will be effectively increased the gas permeability due to more effectively insert between polymer chains of the matrix. The addition of $1 \mathrm{wt} \%$ of modified carbon nanotubes loading to PES resulted in about $64.3 \%$ increases in permeability of $\mathrm{CH} 4$. However, for the small gas molecules such as $\mathrm{CO} 2$ permeability slightly increased with the addition of carbon nanotubes in the PES host matrix. The main pathways of gas transport through the mixed matrix membranes are through dense layer of PES matrix, highly selective carbon nanotubes and non-selective 
gaps or voids between the matrix and sieve particles. From FESEM data in Figure 1-3, the carbon nanotubes are well dispersed in polymer matrix and serve as channels to transport gas molecules. The permeability of $\mathrm{CH} 4$ exhibits the higher increasing of permeability compared to $\mathrm{CO} 2$ gas. The results are consistent with the previous study done by Chen and Sholl [12]. They observed that the main factor affecting the increase of $\mathrm{CH} 4$ permeability with addition of carbon nanotubes into polymer host is due to the extremely fast diffusion of gas molecules adsorbed inside carbon nanotubes. They also reported that the SWNT membranes should be strongly selective for $\mathrm{CH} 4$ and would produce very large fluxes of $\mathrm{CH} 4$. Therefore, as presented in Table 2, the ideal selectivity for $\mathrm{CO} 2 / \mathrm{CH} 4$ of the PES-1 wt\% carbon nanotubes was slightly increased due to preferential sorption of $\mathrm{CH} 4$ in the carbon nanotubes. Moreover, the permeability for unmodified carbon nanotubes for all gases also increases compared to neat PES membrane. However, the $\mathrm{CO} 2 / \mathrm{CH} 4$ selectivity was decreased for unmodified carbon nanotubes mixed matrix membrane. Therefore, the increase of permeability in the PES-unmodified carbon nanotubes was not due to the gas transportation inside the nanotubes. As shown in Figure 2, the PES- unmodified carbon nanotubes are not well distributed in polymer matrix and the severe unselective voids between carbon nano-particles and polymer matrix was appeared. Hence, the PES chains could not fall onto the carbon nanotubes walls tightly and forming a narrow gaps surrounding the carbon nanotubes. Therefore, gas molecules can easily passed through the unselective voids or the gaps. The unselective voids would be functioned as pinholes that allow all gases molecules pass rapidly without any selectivity. Thus, the permeability of all gases is increased thus reducing the gas selectivity. As can be seen in Table 2, the silane treated of modified carbon nanotubes filled PES polymer host mixed matrix membrane improved the $\mathrm{CO}_{2} / \mathrm{CH}_{4}$ selectivities or biogas purification.

\section{CONCLUSION}

In this study, the polyethersulfone (PES) with silanization of carbon nanotubes using Dynasylan Ameo silane coupling agent for the use of fabricating mixed matrix membrane for biogas purification. Based on the experimental results and analysis, the following conclusions can be made.
The FESEM for the cross-sectional and surface area images of mixed matrix membrane films indicated that the modified of carbon nanotubes dispersed well in the polymer matrix. The surface ruptures are not occurred on the carbon nanotubes mixed matrix membrane might be due to the shape of carbon nanotubes appeared to be oblong. The smooth surface of carbon nanotubes might also help to enhance the adherence between the nanotubes and the host polymer.

The PES-modified carbon nanotubes membranes had increased the permeability of methane gas and the $\mathrm{CO}_{2} / \mathrm{CH}_{4}$ selectivity.

The carbon nanotubes have been potentially as in organic filler for mixed matrix membrane for the future biogas purification membrane.

\section{REFERENCES}

[1] Kapdi, S.S., V.K. Vijay, S.K. Rajesh, R. Prasad., Biogas scrubbing, compression and storage: perspective and prospectus in Indian context, Renew. Energy 30 (2005) 1195-1202.

[2] Lin, W.H., and T.S.Chung.. Gas permeability, diffusivity, solubility, and aging characteristics of 6FDA-durene polyimide membranes, Journal of Membrane Science, 186 (2001), 183-193

[3] Baker, R.W., Membrane Technology and Applications, McGraw-Hill, New York,

[4] Hacarlioglu, P., L. Toppare, L. Yilmaz, Polycarbonate-polypyrrole mixed matrix gas separation membranes. J. Membr. Sci., 225. (2003). 51-62.

[5] C.M. Zimmerman, A. Singh., W.J. Koros, Tailoring mixed matrix composite membranes for gas separations. J. Membr. Sci., 137. (1997). 145-154.

[6] T.D. Kusworo, A.F. Ismail, A. Mustafa and T. Matsuura, Dependence of Membrane Morphology and Performance on Preparation Conditions: The Shear Rate Effect in Membrane Casting, Separation and Purification Technology, 61. (2007) 249-257.

[7] S. Kim, T.W. Pechar, E. Marand, Poly(imide siloxane) and carbon nanotube mixed matrix membranes for gas separation, Desalination, 192 (2006) 330-339.

[8] P.M. Ajayan, O. Stephen, C. Colliex, D. Trauth, Science, 265, (1994) 1212-14.

[9] K.-T. Lau and D. Hui, The revolutionary creation of new advanced materials-carbon nanotubes composites, Composites: Part B, 33 (2002) 263-277.

[10] P. C. Ma, J.-K. Kim, B. Z. Tang, Effects of silane functionalization on the properties of carbon nanotube/epoxy nanocomposites, Composites Science and Technology, 67, (2007) 2965-2972.

[11] B.-K. Zhu, S.-H. Xie, Z.-K. Xu, Y.-Y. Xu, Preparation and properties of the polyimide/multi-walled carbon nanotubes (MWNTs) nanocomposites, Comp. Sci. Technol. 66 (2006) 548-554.

[12] H. Chen, D.S. Sholl, Prediction of selectivity and flux for $\mathrm{CH} 4 / \mathrm{H} 2$ separations using single walled carbon nanotubes as membranes, J. Membr. Sci., 269 (2006) 152-160. 